Two other project themes are at an early planning stage:

- Food Safety

- Traffic.

\section{Call for project ideas}

In March 2001 the Board of Technology sent a letter to 200 organisations, educational establishments, private technology enterprises and civil service to invite them to propose ideas for parliamentary technology assessment projects. The idea behind the invitation was to pick up suggestions for technology assessment projects which needed more public elaboration and attention. The Board will make this a yearly tradition if it proves to be valuable.

\section{Web}

The Norwegian Board of Technology has a website (www.teknologiradet.no), but it is mainly in Norwegian. An English version is under construction.

\section{Contact}

Kjetil K. Jasund

The Norwegian Board of Technology

Box 522 Sentrum, 0105 Oslo, Norway

Tel.: + 4723318300

Fax: + 4723318301

e-mail: post@teknologiradet.no

\section{$\gg$}

\section{Technikbewertung in der Lehre}

von Michael F. Jischa, Technische Universität Clausthal

Dies ist ein Erfahrungsbericht über einen erfolgreichen Prozess, das Fach Technikbewertung in die Ingenieurwissenschaften einzuführen. Erfahrungsberichte sind notwendigerweise subjektiv. Von daher können sie keine Blaupause für eine direkte Übertragung in andere Umfelder sein, wenngleich die hier vorgetragene Vorgehensweise durchaus exemplarischen Charakter haben kann.

\section{Die Voraussetzungen}

Einleitend beginne ich mit der stets zentralen Frage, wer was warum wann und wo fragt. Wenn diese Elemente bruchlos aufeinander aufbauend stimmig und glaubwürdig erscheinen, so ist eine wesentliche Voraussetzung für den Erfolg gegeben. Der Rest ist persönliches Engagement, strategisches Gespür und die Bereitschaft zur Übernahme zusätzlicher Verpflichtungen, zumindest in der Startphase.

Wer: Der Autor ist Ingenieur mit Erfahrungen in Strömungsmechanik, Thermodynamik und Allgemeiner Mechanik, also den wesentlichen strukturellen Grundlagen der Ingenieurwissenschaften, und im Management. Insgesamt gute Voraussetzungen für einen Start in Neuland. Ingenieur zu sein bedeutet, proble mlösungsorientiert $\mathrm{zu}$ arbeiten. Das erleichtert die Einführung neuer Lehrinhalte in die Studienpläne. Beispielhaft seien Informationstechnik und Qualitätsmanagement genannt, die erst in jüngerer Zeit in Lehrpläne aufgenommen wurden. Hier ist das pragmatische Wissenschaftsverständnis der Ingenieure sicher hilfreich, während das wenig ausgeprägte Selbstreflexionsvermögen der Ingenieure den Blick für das Erkennen von nichttechnischen Problemen häufig versperrt.

Was: „Die ökologische Wende“ der sechziger Jahre (von Lersner 1992) markiert den Beginn des Problembewusstseins. Die Diskussionen über TA (Technology Assessment) und über die "Grenzen des Wachstums" (Meadows 1972) als ersten Bericht an den 1968 gegründeten Club of Rome haben in dieser Zeit bgonnen und sind mit dem allseits akzeptierten Leitbild Nachhaltigkeit eng verknüpft. Dies wird in einem ersten Schritt in einer Sensibilisierungsvorlesung herausgearbeitet.

Warum: Zur Charakterisierung der Umweltpolitik hat Meyer-Abich (1988) den Dreisatz formuliert: „1.) So geht es nicht weiter. 2.) Was statt dessen geschehen müßte, ist im wesentlichen bekannt. 3.) Trotzdem geschieht es im wesentlichen - nicht." In einer Sensibilisierungsvorlesung „Herausforderung Zukunft" (Jischa 1993) behandele ich die Punkte 1 und 2. Der Punkt 3 ist Anlass für den zweiten Schritt gewesen, die Operationalisierungsvorlesung „Technikbewertung“" zu konzipieren.

Wann: Die Sensibilisierungsvorlesung „Herausforderung Zukunft" fand erstmalig im 
WS 91/92 statt. Genau dieser Zeitpunkt hat sich als Glücksfall erwiesen, weil er in die Vorbereitungen zur Rio-Konferenz für Umwelt und Entwicklung 1992 fiel. So konnte ich mïhelos auf viele aktuelle Berichterstattungen in der Presse zurückgreifen, was der Attraktivität und Akzeptanz der ersten Vorlesung zugute kam.

Wo: Neben dem richtigen Zeitpunkt war der richtige Ort überaus hilfreich. Hierfür bot die TU Clausthal hervorragende Voraussetzungen: Kleiner Ort; überschaubares und fast familiäres Umfeld mit etwa 3000 Studenten und knapp 100 Professoren; kaum großstadtübliche Ablenkungen und daher große Bereitschaft, auch freiwillig Abendveranstaltungen zu besuchen; Lehrangebot überwiegend in Natur- und Ingenieurwissenschaften und somit eine hohe Bereitschaft der Studenten, über den Tellerrand der eigenen Disziplin blicken zu wollen. Diese einleitenden Bemerkungen habe ich bewusst ausführlich gehalten. Denn ein Feldherr muss Fortune haben, wie Napoleon gesagt hat.

\section{Aufbau der Vorlesungen: bottom up}

Nunmehr möchte ich die Inhalte der drei Vorlesungen kurz skizzieren, die ich zunächst im studium generale angeboten habe. Damit kein Missverständnis aufkommt: Zusätzlich zu meinen normalen Lehrverpflichtungen im Institut für Technische Mechanik, sozusagen als Kür neben der Pflicht.

1. Schritt: Sensibilisierungsvorlesung „Herausforderung Zukunft", erstmalig gehalten im Wintersemester 1991/92 und wie folgt gegliedert: 1. Menschheitsgeschichte und Umwelt, 2. Wachstum und Rückkopplung, 3. Bevölkerungsdynamik, 4. Energie, 5. Treibhauseffekt und Ozonloch, 6. Unsere Umwelt, 7. Endliche Ressourcen, 8. Die Dritte Welt, 9. Technik und Ethik, 10. Modelle und Prognosen, 11. Wer kann was tun? Die drängende Nachfrage der studentischen wie auch der externen Hörer nach Vorlesungsunterlagen führte $\mathrm{zu}$ dem gleichnamigen Buch (Jischa 1993). Aus dieser Sensibilisierungsvorlesung sind bislang zwei weitere Vorlesungen entstanden.

2. Schritt: Operationalisierungsvorlesung „Technikbewertung“: Ausgehend von dem
Kapitel „Technik und Ethik“, in dem auf die VDI-Richtlinie Technikbewertung und auf die Geschichte der TA-Entwicklung eingegangen wird, haben B. Ludwig und der Autor gemeinsam eine Operationalisierungsvorlesung mit dem Titel „Technikbewertung" konzipiert und diese erstmalig im Wintersemester 1994/95 angeboten. Deren Gliederung sei kurz vorgestellt (Skript $\mathbf{J}-$ scha, Ludwig). In einer ausführlichen Einleitung werden thematisiert: Warum brauchen wir Technikbewertung als Zusammenfassung der „Herausforderung Zukunft" (Jischa 1993). Danach wird die Frage nach der Verantwortung für Technik alsammen mit dem Leitbild Nachhaltigkeit behandelt. Alsdann werden die historische Entwicklung und der Diskussionsstand von TA, TA-durchführende Institutionen sowie die VDI-Richtlinie Technikbewertung (VDI 1991) besprochen. Es folgt ein Abriss der Systemtheorie, weil aus Ingenieurssicht der Zusammenhang von Technikbewertung und Systemanalyse naheliegend ist. Daran schließt sich die Besprechung publizierter TA-Studien an, die nach zwei Kriterien ausgewählt wurden: Relevanz des Themas und saubere Herausarbeitung der gewählten Methode(n). Zwei gute Beispiele für die Herausarbeitung der gewählten Methoden sind aus unserer Sicht der Deutsche Delphi-Bericht zur Entwicklung von Wissenschaft und Technik, bearbeitet vom Fraunhofer-Institut für Systemtechnik und Innovationsforschung (ISI 1993), in Bezug auf die Delphi-Methode, sowie die Prognosstudie Technikfolgen Chlorchemie (Prognos 1994) bezüglich der Szenariotechnik. Aus Aktualitätsgründen behandeln wir die TA-Studien zum Raumtransportsystem SÄNGER des Büros für Technikfolgen-Abschätzung beim Deutschen Bundestag (TAB; Paschen u. a. 1992), zum Transrapid bzw. Hochleistungsschnellverkehrssystem (Battelle 1976) sowie zur Solarenergienutzung (Jochem 1988). Die Vorlesung wird durch eine zusammenfassende Behandlung von weiteren Methoden sowie von Instrumenten (Ökobilanz, Produktlinienanalyse, Umweltverträglichkeitsprüfung, Umweltaudit, Ökocontrolling, 
Umweltinformations- und Umweltmanagementsysteme) abgeschlossen.

3. Schritt: Die Anschlussvorlesung „Dynamische Systeme in Natur, Technik und Gesellschaft“ ist aus dem Kapitel „Modelle und Prognosen" (siehe 1) und aus dem Kapitel „Systemtheorie“ (siehe 2) hervorgegangen (Skript Jischa). Sie verfolgt zwei Ziele. Zum einen soll sie den Studenten die Gemeinsamkeiten dynamischer Systeme unterschiedlicher Herkunft aufzeigen. Zum anderen soll sie der Kritik von Kollegen begegnen, ich sei in die weichen Disziplinen abgedriftet. Daher wähle ich den Begriff Anschluss, um meine Verbindung $\mathrm{zu}$ dem üblichen Tun von Ingenieuren zu verdeutlichen. Dynamische Systeme in der Technik (Schwingungen, Chemische Reaktoren, Wellenausbreitung in Strömungen, Bénard-Konvektion, Verkehrsdynamik) habe ich durch Modelle aus der Ökosystemforschung (Räuber/Beute-, Konkurrenz-, Symbiose-, Populationsdynamik sowie Überfischung, Epidemie- und Diffus ionsmodelle) und Modelle in der Gesellschaft (Beispiele aus Verhaltensdynamik, Volkswirtschaftslehre, Zivilisationsdynamik und Ökolopoly nach Vester) quasi eingerahmt. Computersimulationen in begle itenden Übungen erhöhen die Attraktivität zusätzlich. Es lässt sich spannend vermitteln, dass das Problem der Überfischung und die Stabilität eines chemischen Reaktors mathematisch ähnlich sind. Hinzu kommt, dass Themen zum deterministischen Chaos bei mathematisch hinreichend belastbaren Studenten der Natur- und Ingenieurwissenschaften generell auf großes Interesse stoßen.

Die hervorragende Akzeptanz der drei Vorlesungen hat dazu geführt, dass diese nunmehr als Pflichtfächer an der TU Clausthal eingeführt wurden: „Herausforderung Zukunft“ als „Grundlagen des Umweltschutzes“ im Grundstudium des neuen Studienganges Umweltschutztechnik; ,Technikbewertung“ im Hauptstudium der Studiengänge/-richtungen Umweltschutztechnik, Energiesystemtechnik, Rohstoff- und Geotechnik sowie Wirtschaftsingenieurwesen; „Dynamische Systeme in Natur, Technik und Gesellschaft" im Hauptstudium des Studiengangs Energiesystemtechnik. Die aufgeführten Vorlesungen werden von einer wachsenden Zahl von Studenten anderer Studiengänge als Wahlfächer gewählt.

Diese überaus positive Entwicklung eines bottom-up Ansatzes (einfach tun) wurde durch studentische Forderungen ebenso unterstützt wie durch weitsichtige Kollegen. Weitere flankierende Maßnahmen waren zweifellos überaus hilfreich, weswegen ich diese kurz skizzieren möchte. Hiermit meine ich das Forum Clausthal sowie die Evangelische Studentengemende Clausthal (ESG), eigene Forschungsarbeiten und Projekte sowie Aktivitäten außerhalb der TU Clausthal.

\section{Das Forum Clausthal}

Im Wintersemester 91/92 hat der damalige Studentenpfarrer K. Wachlin die Idee einer interdisziplinären Arbeitsgruppe Forum Clausthal in unsere Universität hineingetragen. Der seinerzeitige Rektor Prof. G. Müller hat diese Idee aufgegriffen und mich mit dessen Weiterverfolgung beauftragt, offenbar weil genau zu diesem Zeitpunkt meine erste Sensibilisierungsvorlesung mit starker Resonanz lief. Wachlin und der Autor begannen, die Idee Forum Clausthal mit Leben zu erfüllen. Die erste Seminarveranstaltung trug 1992 den Titel „Technikbewertung - eine interdisziplinäre Aufgabe“. Es folgten Veranstaltungen zu Themen wie Energiepolitik und $\mathrm{CO}_{2}$-Minderungspotentialen; zu Sustainable Development (über zwei Semester hinweg); zur Frage, was Ingenieure und Naturwissenschaftler der Zukunft können müssen; zu Verkehrskonzepten, zur Informationsgesellschaft, zu nuklearen Entsorgungskonzepten, zur Konfliktkommunikation und zum Produktdesign. Die beiden letzten Veranstaltungen waren den Themen „Die Zwei Kulturen" sowie „Kunststoffe und Umwelt" gewidmet. Nahezu alle Beiträge sind in einer Schriftenreihe dokumentiert. Forum Clausthal ist mittlerweile etabliert, es gehört zu dem ersten Schritt, der Sensibilisierungsphase.

Nach dem Weggang von K. Wachlin zur Akademie für Technikfolgenabschätzung in Baden-Württemberg, Stuttgart, haben wir von Seiten der TU Clausthal die Verantwortlichen der Ev.-luth. Landeskirche Hannovers erfolgreich davon überzeugen können, dass die aus Einspargründen gefährdete Wiederbesetzung 
der Stelle des Studentenpfarrers nachdrücklich von uns gewünscht wird. Wir haben daran die Erwartungshaltung geknüpft, dass ein junger, engagierter und möglichst promovierter Theologe eine große Chance darin sieht, mit Studenten der Natur- und Ingenieurwissenschaften $\mathrm{zu}$ kommunizieren. Bei entsprechender Neigung und Qualifikation hatten wir die Erteilung eines Lehrauftrages in Aussicht gestellt.

All dies hat sich mehr als erfüllt. Der amtierende Studentenpfarrer M. Schlicht, in Kirchengeschichte promoviert, nimmt nun schon seit sieben Semestern einen Lehrauftrag „Wissenschaft, Technik und Ethik“"wahr. Die Vorlesung wird hervorragend angenommen, und sie wird durch eine außerordentlich lebendige Seminarreihe in der ESG mit Vorträgen aus den Bereichen Wissenschaft, Wirtschaft, Gesellschaft und Politik wirkungsvoll ergänzt. Diese Aktivitäten dienen sowohl der Sensibilisierung (1. Schritt), der Ergänzung der Operationalisierung Technikbewertung (2. Schritt) sowie dem Anschluss (3. Schritt) an andere Disziplinen.

\section{Forschung anhand praxisrelevanter Frage- stellungen}

Lehre ohne begleitende Forschung kann nicht überzeugen. Aus diesem Grund habe ich nach TA-relevanten Forschungsthemen gesucht. Dabei habe ich mich zunächst stark an die VDI-Richtlinie Technikbewertung (1991) angelehnt, die aus meiner Sicht als Ingenieur einen Glücksfall darstellt. Ebenso wichtig war die Suche nach mutigen Doktoranden. Mein erster TA-Doktorand war B. Ludwig, den ich glücklicherweise davon überzeugen konnte, statt einer angedachten Arbeit über die Modellierung einer Zweiphasenströmung auf der Basis einer Zweiphasen-Kontinuumstheorie es doch gleich mit noch komplexeren Systemen $\mathrm{zu}$ versuchen. Er ließ sich darauf ein, wobei unser beiderseitiges Einarbeiten in die für uns neue Problematik zu der gemeinsamen Konzipierung der Vorlesung „Technikbewertung“ führte.

Die Dissertation „Methoden zur Modellbildung in der Technikbewertung" von Ludwig (1995) wirkte wie eine Initialzündung. Es folgten die Dissertationen ,Zustandsbeschreibung und Dynamik umweltrelevanter Systeme“
(Tulbure 1997), „Umweltmanagementsysteme bei Dienstleistungsunternehmen“ (Kensy 1998), „Quantifizierung nachhaltiger motorisierter Mobilität“ (Volkmar 1999) und „Nachhaltigkeit im Verkehrsbereich durch netzgestützte kooperative Planungs- und Entscheidungsunterstützung“ (Decker 2000). Diese Dissertationen sind in der CUTECSchriftenreihe der Clausthaler UmwelttechnikInstitut $\mathrm{GmbH}$ erschienen.

$\mathrm{Zu}$ den genannten internen Dissertationen sind externe Dissertationen hinzu gekommen. Aufgeführt seien hier eine Arbeit zu „Life Cycle Assessment of Natural Gas Vehicles“ (Nigge 2000), die in der Schriftenreihe der Europäischen Akademie bei Springer erschienen ist. Zwei weitere stehen kurz vor dem Abschluss: „Entwicklung eines integrierten Modells zur Bewertung von Maßnahmen im Straßenverkehr“ sowie „Indikatorengestützte Umweltbewertung zur Steuerung der Produktentwicklung in der Automobilindustrie", beide Volkswagen AG. Die Vorarbeiten von derzeit vier weiteren Doktoranden sehen erfolgversprechend aus.

Weiter möchte ich ein Forschungsprojekt, finanziert von der Hanns-Lilje-Stiftung zum Thema „Operationalisierung des Leitbildes Nachhaltige Entwicklung durch Technikbewertung" schildern. Dies wird von C. Berg, Physiker und Theologe, bearbeitet. Zielvorstellung ist eine zweifache Promotion, sozusagen als Blick aus zwei verschiedenen (Wissenschafts-) Richtungen. Berg hat soeben seine theologische Dissertation zum Verhältnis von Theologie zu Naturwissenschaft und Technik an der Universität Heidelberg eingereicht. Sein daran anknüpfendes Clausthaler Dissertationsthema ist in Umrissen skizziert.

Ausgehend von meiner These, TA als Operationalisierung des diffusen Leitbildes Nachhaltigkeit zu verstehen, resultiert Forschungsbedarf in folgenden Feldern:

- Zustandsbeschreibung durch Nachhaltigkeits indikatoren

- Umgang mit unsicherem, unscharfem sowie Nicht-Wissen

- (Weiter-) Entwicklung von Methoden und Instrumenten

- Orientierung an Werten und Umgang mit Wertkonflikten

- Simulation dynamischer Systeme. 
Vertreter anderer wissenschaftlicher Disziplinen werden zu anderen Forschungsthemen und Prioritäten kommen, daran liegt ja gerade der Reiz des TA-Konzeptes.

Meine Sichtweise ist die eines Ingenieurs, eines Problemlösers. Eine Promotion in einer ingenieurwissenschaftlichen Fakultät ist typischerweise wie folgt strukturiert: Was ist das Problem? Warum ist es ein Problem? Was haben andere zur Problemlösung getan? Was hat der Doktorand zur Problemlösung beigetragen?

Alle abgeschlossenen und laufenden Promotionsvorhaben lassen sich den fünf genannten Feldern, teilweise überlappend, zuordnen. Die Behandlung erfolgt stets anhand konkreter, praxisrelevanter Fragestellungen. Da eine Zlordnung zu etablierten akademischen Disziplinen (ist es Maschinenbau, Energie- und Verfahrenstechnik, Informatik oder Betriebswirtschaftslehre?) bei den skizzierten Themen kaum möglich ist, legen wir auf Interdisziplinarität durch Auswahl der Gutachter Wert. Neben Ingenieuren waren bislang Informatiker, Betriebs- und Volkswirte sowie Philosophen als Gutachter eingebunden.

Letzteres gilt ebenso für die erste Habilitation in dieser neuen Disziplin, wobei wir aus verschiedenen Gründen den einerseits etablierten, aber gleichwohl hinreichend unscharfen Begriff Systemtechnik (und nicht TA) gewählt haben. Eine Habilitationsschrift zu „Management komplexer Systeme" wurde abgeschlossen und erscheint in Kürze (Ludwig 2000). Ein weiteres Habilitationsverfahren wird in etwa einem Jahr eingeleitet.

Mir erschienen diese ausführlichen Bemerkungen zu flankierenden Forschungsarbe iten, aus denen zahlreiche Studien- und Diplomarbeiten hervorgegangen sind, absolut notwendig zu sein. Denn wenn ein Fach wie TA dauerhaft etabliert werden soll, dann muss der Forschungsbedarf eindeutig beschrieben werden können. Wenn darüber hinaus, wie in der Clausthaler Situation, auch Drittmittel eingeworben werden, entfällt ein weiterer Kritikpunkt von potenziellen Bedenkenträgern und Verhinderern (,Es könnte ja nur Feuilleton sein").

Als letzte flankierende Maßnahme möchte ich Aktivitäten außerhalb meiner Lehr- und Forschungstätigkeit im Institut für Technische Mechanik erwähnen. Diese beinhalten ein gro- ßes Synergiepotential. Hier möchte ich folgende Funktionen/Mitgliedschaften nennen: Präsident der Deutschen Gesellschaft Club of Rome, Mitglied im Kuratorium der Hanns-LiljeStiftung, Mitglied in der Bereichsvertretung „Mensch und Technik“ sowie „Technikbewertung" des VDI, Sprecher der interdisziplinären Arbeitsgruppe Forum Clausthal sowie Vorsitzender des Hochschulbeirats der Clausthaler Umwelttechnik-Institut GmbH (CUTEC).

Das geschilderte Bündel von Aktivitäten hat dazu geführt, dass (zumindest derzeit) das Fach Technikbewertung an der TU Clausthal etabliert ist. Interne Strukturüberlegungen laben eingesetzt, seit ich meine vorzeitige Emeritierung zum März 2002 beantragt habe. Es erscheint denkbar, durch Einrichtung einer Professur für Systemtechnik (als Arbeitstitel) für eine Verstetigung zu sorgen. Dieser Prozess ist jedoch kein Selbstläufer. Denn angesichts von Einsparauflagen und latent vorhandenen kollegialen Bedenken gegen derartige Fächer, die (noch) nicht zu dem gehören, was man gern die Kernkompetenz von Ingenieuren nennt, sind die Erfolgsaussichten offen.

Glücklicherweise gibt es unterstützende Stellungnahmen des VDI: Ingenieurausbildung im Umbruch - Empfehlung des VDI für eine zukunftsorientierte Ingenieurqualifikation (1995) sowie Memorandum des VDI zum Wandel des Ingenieurberufsbildes (1997). Darin heißt es z. B.: ,Zum modernen Grundlagenwissen gehören nach Meinung des VDI auch ökologische Kenntnisse im Anwendungszusammenhang der jeweiligen Technologie und Kenntnisse über Inhalte und Verfahren der Technikbewertung“(VDI 1995).

\section{Fazit}

Lassen Sie mich ein abschließendes Fazit zu der Überschrift „TA in der Lehre“ ziehen. Das von uns entwickelte Vorlesungskonzept ist mehrfach getestet worden. So kontinuierlich seit 1994/95 an der TU Clausthal wie auch extern: An der TU Chemnitz (Jischa, Ludwig) und an der FH Wolfenbüttel (Lehraufträge für Ludwig und für Frau Tulbure) sowie durch zahlreiche Vorträge. Wir sind dabei, die bisher beispielhaft vorgestellten Studien kontinuierlich zu ergänzen, um adressatenorientiert auf verschiedene Interessenlagen eingehen zu kön- 
nen. Dafür kommen nach meinem derzeitigen Kenntnisstand in Frage: Die Delphi-Befragung 1996/98 über Potenziale und Dimensionen der Wissensgesellschaft (Prognos für das BMBF); PVC und Nachhaltigkeit (1999, Prognos für die AG PVC und Umwelt); die TAB-Berichte zur Verkehrssituation sowie zur Forschungs- und Technologiepolitik für eine nachhaltige Entwicklung. Mit Spannung erwarte ich das in Arbeit befindliche HGF-Projekt ,Global zukunftsfähige Entwicklung - Perspektiven für Deutschland“, das wir ebenfalls für die Vorksung aufbereiten wollen.

Ich möchte mein Fazit mit der Anregung beenden, weitere TA-Lehrkonzepte vorstellen zu lassen. Beispielhaft nenne ich K. A. Detzer (Lehrauftrag an der TU München) und stellvertretend für weitere Kollegen an der FH Hamburg A. von Gleich (siehe hierzu VDI Report 28).

Armin Grunwald (ITAS) hat sein Konzept, dem Lehrstuhl ,Technikfolgenabschätzung und Systemanalyse“ an der Universität Freiburg Kontur zu verleihen, in den TADatenbank-Nachichten (Heft 3, Oktober 2000, S. 139-140) vorgestellt: Eine Vorlesung „Einführung in die Technikfolgenabschätzung“" wird durch ein Seminar „Verantwortung von Ingenieuren und Naturwissenschaftlern" ergänzt. Ich bin auf einen späteren Erfahrungsbericht nach ersten Probeläufen mit (hoffentlich!) guter Resonanz gespannt.

Eines sollten wir von den verschiedenen Lehrkonzepten nicht erwarten: Dass wir uns auf einen einheitlichen Lehrkanon einigen könnten. Dies ist weder möglich noch wünschenswert. Gerade in der Vielfalt der Lehrinhalte und Lehrformen liegt der Reiz der TADisziplin. Wir sollten dies als eine Chance begreifen, die Gräben zwischen den ,zwei Kulturen“, den Natur- und Ingenieurwissenschaften einerseits sowie den Geistes- und Cesellschaftswissenschaften andererseits, nicht etwa einzuebnen (das wäre fatal), sondern verschiedenartig gestaltbare Brücken zu bauen. Wir können und müssen viel voneinander lernen.

\section{Anmerkungen}

Bezüglich Literaturangaben und auch weiterer $\mathbf{h}$ formationen, insbesondere $\mathrm{zu}$ Forschungsthemen, verweise ich auf meinen Artikel ,Technikfolgenab- schätzung in Lehre und Forschung“ in Petermann und Coenen (Hrsg.), 1999: Technikfolgen-Abschätzung in Deutschland. Frankfurt/Main: Campus. S. 165 - 195.

Das Buch „Herausforderung Zukunft“ ist im Buchhandel nicht mehr erhältlich. Es ist jedoch über unser Institut zu beziehen. Das gilt auch für die Skripte „Technikbewertung“ sowie „Dynamische Systeme in Natur, Technik und Gesellschaft“. Die Hefte der CUTEC-Schriftenreihe sowie der Schriftenreihe FORUM CLAUSTHAL sind in dem Verlag PAPIERFLIEGER, Telemannstr. 1, 38678 Clausthal-Zellerfeld erschienen. Meiner homepage können sie weitere Informationen entnehmen.

\section{Kontakt}

Prof. Dr.-Ing. Michael F. Jischa

Institut für Technische Mechanik der TU Clausthal Graupenstraße 3, D-38678 Clausthal-Zellerfeld

Tel.: + 49 (0) 5323 / 72-20 83

Fax: + 49 (0) 5323 / 72-22 03

E-Mail: jischa@itm.tu-clausthal.de Internet:http://www.itm.tu-clausthal.de/

\section{Die Zukunft der Mobilität ge- stalten. Das neue Fraunhofer- Institut für Verkehrs- und Infrastruktursysteme}

\author{
von Jörg Schütte und Günther Nirschl, \\ Fraunhofer IVI Dresden
}

Die Fraunhofer-Gesellschaft (FhG) sieht die Verkehrstechnik als strategisches Arbeitsfeld. Dieser Bedeutung wurde Anfang 1999 mit der Gründung eines neuen FraunhoferInstituts für Verkehrs- und Infrastruktursysteme (IVI) in Dresden Rechnung getragen. Damit wurde das Angebot der FhG erweitert und konzentriert, zur Steigerung der Leistungsfähigkeit, Sicherheit, Umwelt- und Nutzerfreundlichkeit künftiger Verkehrssy steme durch technologische Lösungen beizutragen.

\section{Zielsetzung}

Das neue Fraunhofer IVI hat das zentrale Ziel, durch geeignete Forschung dazu beizutragen, auch bei begrenzten Infrastrukturressourcen dauerhaft Mobilität zu erhalten. Die Aufgaben 